\title{
Pour une introduction au forum sur le «Printemps arabe»
}

Nader Vahabi

\section{(e) OpenEdition \\ Journals}

Édition électronique

URL : http://journals.openedition.org/conflits/18211

DOI : 10.4000/conflits. 18211

ISSN : $1777-5345$

Éditeur :

CCLS - Centre d'études sur les conflits lilberté et sécurité, L'Harmattan

\section{Édition imprimée}

Date de publication : 30 décembre 2011

Pagination : 101-107

ISBN : 978-2-296-55700-0

ISSN : 1157-996X

Référence électronique

Nader Vahabi, « Pour une introduction au forum sur le «Printemps arabe» », Cultures \& Conflits [En ligne], 83 | Automne 2011, mis en ligne le 04 janvier 2013, consulté le 30 mars 2021. URL : http:// journals.openedition.org/conflits/18211; DOI : https://doi.org/10.4000/conflits.18211 


\section{Pour une introduction au forum sur le «Printemps arabe»}

\section{Nader VAHABI}

Nader Vahabi est spécialiste de l'immigration iranienne, auteur de plusieurs livres et articles, chercheur associé à deux centres de recherche : le CADIS (Centre d'analyse et d'intervention sociologiques) de l'École des hautes études en sciences sociales (EHESS), Paris et le CEDEM (Centre d'études de l'ethnicité et des migrations) de l'Université de Liège, Belgique. Il dirige à Paris le CEDIR, Centre d'études sur la diaspora iranienne. Son dernier livre, La migration iranienne en Belgique, Une diaspora par défaut, est paru chez l'Harmattan en mai 2011.

$\mathrm{O}$ $\mathrm{n}$ dit souvent que des irruptions d'événements inattendus et le génie de l'histoire se manifestent là où nul ne les attend. Plus de 330 millions d'hommes et de femmes, vingt et un pays, une grande partie d'un continent ont été touchés par une tempête irrépressible, relayée par le virtuel dans ses différentes formes, surtout par les chaînes de télévision par satellite, et personne ne sait où elle pourra encore frapper. Révolutions ou révoltes nous interpellent : en arabe, les deux mots se disent «Thawra » qui signifie révolution, ce que l'on entendait dans la rue ${ }^{1}$. Ce mot est-il justifié ?

Dans son étude sur les phases déterminantes que comporte " naturellement » une révolution, Crane Brinton ${ }^{2}$, se fondant sur les quatre " grandes révolutions », dégage des étapes-clés. Comparant les révolutions anglaise, américaine, française et russe et cherchant à faire ressortir les « uniformités » de ces épisodes historiques, il distingue des périodes communes à toutes les révolutions nationales ${ }^{3}$.

1. Laurens H., historien, entretien avec le journal Libération, «Le printemps des rêves arabes ", Hors-série, p. 43.

2. Crane Brinton C., The anatomy of revolution, New York, Vintage Books, 1965 (1ère édition 1938 ), pp. 132-146.

3. Pour une approche critique de C. Brinton, voir Dobry M., Sociologie des crises politiques, La dynamique des mobilisations multisectorielles, Paris, Presses de la fondation nationale des sciences politiques, 1992, pp. 60-64. 
De la première phase, celle de la prise du pouvoir « quand ceux d'en bas ne veulent plus être gouvernés et quand ceux d'en haut ne peuvent plus gouverner ", marquée par l'effervescence révolutionnaire, on passe à la phase de la mise à l'écart des modérés et du « règne des radicaux ». Arrive alors la troisième phase dans laquelle on assiste à l'émergence d'un homme fort menant une terreur inédite avec un groupe restreint. La quatrième étape, dite "thermidorienne", se caractérise par un retour à un statu quo supportable, la société établissant un équilibre.

Nonobstant la simplification excessive inhérente à toute modélisation que sous-tend une démarche d' "histoire naturelle" 4 , ce schéma nous oriente vers les logiques, les piliers, les traits communs marquant les révolutions tunisienne et égyptienne.

Pour le cas tunisien, les événements se situant aux limites de l'émeute et de la révolution qui se sont déroulés en un temps très condensé, depuis l'immolation d'un jeune homme le 17 décembre 2010 jusqu'à la chute de Ben Ali le 14 janvier 2011 - soit moins d'un mois - ont provoqué un véritable état de sidération dans le monde. De même, en Égypte, dix-sept jours seulement se sont écoulés depuis la première manifestation, le 25 janvier 2011, jusqu’à la démission de Moubarak, le 11 février 2011. Ces deux révolutions-éclairs 5 revêtent ainsi des caractères d' "outsiders » qui ont sidéré un grand nombre de personnes quoique la « décapitation » des seuls chefs du palais n'eût pas touché l'ensemble du système.

L'état de sidération provient du fait que ces événements, selon tous les témoignages, étaient imprévisibles et ont surgi là où on était loin de les attendre. L'ambassadeur de France à Tunis lui-même, comme la ministre des Affaires étrangères estimaient en décembre 2010 que le président Ben Ali était inamovible. Des faits analogues sont repérables dans un grand nombre d'épisodes révolutionnaires : on ne sait jamais quelle est l'étincelle qui mettra le feu aux poudres, car l'apathie des masses donne souvent une fausse impression de stabilité qui cache en fait des mouvements de fond affectant en premier lieu la jeunesse ${ }^{6}$.

Toutefois, certaines différences s'imposent qui font des deux révolutions tunisienne et égyptienne des «outsiders » par rapport aux autres révolutions : elles revêtent en effet des caractères spécifiques liés, d’une part, à leur réussite

4. Dobry M., Sociologie des crises politiques, op. cit., p. 63.

5. Pour les chronologies de ces deux révolutions, $c f$. "Comprendre le réveil arabe », Manière de voir, Le Monde diplomatique, ${ }^{\circ} 117$, bimestriel, juin-juillet 2011. Cf. aussi, « Les printemps des rêves arabes ", Libération, hors-série, été 2011.

6. Il est intéressant de signaler qu'en août 1978 la CIA (Central Intelligence Agency) dans une analyse de la situation de l'Iran a conclu : « l'Iran n'est pas dans une situation révolutionnaire ni même pré-révolutionnaire » Taruk Tazdaït et Nacer Chaabane, « De quelques caractéristiques des révolutions ", Manière de voir, n¹17, pp. 36-38. 
dans un laps de temps très court et, d'autre part, à leur vision sécularisée de l'islam.

Ce rapide état des lieux concernant les deux révolutions, associé à une étude exploratoire de juillet/août 2011, nous a fait envisager, dans le cadre d'un forum pour ce numéro de Cultures $\mathcal{E}$ Conflits, de solliciter différents spécialistes de la question afin de préparer des textes courts apportant un éclairage particulier sur ces épisodes révolutionnaires. Huit angles d'attaque, présentés ci-dessous, étaient alors envisageables.

\section{Définition et nature de la révolution}

Si l'on tient compte des grandes révolutions classiques, peut-on qualifier les mouvements tunisien et égyptien de révolutions ou faudrait-il leur conférer une autre définition ? Le terme révolution est-il adéquat alors que les fondements du pouvoir ne basculent pas ? Peut-on établir un parallélisme avec le Printemps des peuples européens des années 1848-49 qui s'est terminé dans les glaces de la réaction la plus terrible ? Les deux mouvements sont-ils comparables aux révolutions des pays de l'Europe de l'Est en 1989 ? Ils insistent en effet sur l'instauration d'une légitimité démocratique et évoquent leur référence aux droits de l'homme et à la liberté qui garantit un système politique pluraliste issu d'élections libres excluant la notion du leader à vie. La rupture avec l'autoritarisme ne constituerait-elle pas à elle seule une avancée historique dans un monde où les autocrates semblent inébranlables, d'autant plus que les révolutions tunisienne et égyptienne présentent la remarquable particularité de l'absence de leaders reconnus?

Dans ces conditions, on s'intéresse plus que jamais au sens de la révolution et à son dynamisme socio-historique puisque, depuis la chute du mur de Berlin, la notion a été complètement transformée, ce qui demande une étude de ces modifications.

\section{Les similarités et les divergences du monde arabe}

Qu'entendons-nous par le monde arabe ? Quelles sont les caractéristiques politiques, ethniques, linguistiques et religieuses des sociétés arabes ? Pourquoi leurs similitudes mettent-elles en exergue des valeurs négatives : la corruption, le despotisme, le manque de liberté d'expression et le manque de justice sociale ? Ces aspects négatifs sont-ils à l'origine du printemps arabe ?

Le texte de N. Messari, "Au-delà des clichés : différences et similarités entre les pays arabes ", aborde ces thématiques. D’après l'auteur, du golfe Persique à l'océan Atlantique, on s'identifie au monde arabe en insistant sur les liens religieux, linguistiques et historiques communs. D'autres similarités existent aussi, sans qu'elles soient forcément négatives : c'est le cas par exem- 
ple pour la pyramide des âges qui présente une base très large indiquant une forte proportion de jeunes, souvent frappés par le chômage cependant. L'auteur admet ces similarités mais, pour lui, elles «ne constituent certainement pas des facteurs d'union entre ces pays, et encore moins des éléments d'homogénéité entre eux ». Il en conclut qu'il y a donc nécessité de rester extrêmement prudent lorsqu'on parle du monde arabe.

\section{Le duo de la transition démocratique et de la justice sociale}

Les deux révolutions sont-elles terminées ? Iront-elles vers un statu quo ? Sont-elles appelées à engendrer une véritable transition démocratique, à être la marée humaine chaleureuse et foisonnante du « démocratisme radical » ou à devenir la froide raison technocratique de la gouvernance mondiale qui garde un œil intéressé sur le pétrole de l'Orient ? On peut poser la question d'une autre manière : peut-on renoncer à la justice pour la liberté ou à la liberté pour la justice ? Sommes-nous à un moment où le social prendrait le pas sur le politique?

Le texte de F. Khosrokhavar, «Les révolutions arabes : révolutions de justice et de liberté », examine ces différentes questions. L'auteur dresse un bref historique de la transition, vers 1980, des économies de certains pays arabes (Tunisie, Égypte) vers un capitalisme libéral, ouverture qu'il qualifie d' "agression ». Il justifie ce jugement en constatant parallèlement la disparition de pans entiers de l'État providence qui aidait les plus démunis. L'auteur craint que le modèle néo-libéral adopté ne laisse inaccessible l'idéal de justice sociale.

\section{Les acteurs, les partis politiques, les syndicats et les jeunes}

Qui sont les acteurs qui mobilisent et impulsent le sens de la révolution en Tunisie ? La « rue » et « la place » ont été le fer de lance du mouvement et sont à l'origine de la mobilisation des jeunes et des réseaux de socialisation des classes moyennes et intellectuelles. Comment, sans disposer d'expérience politique préalable, ont-ils pu assurer la dynamique protestataire du mouvement ? Quelles sont les relations entre, d'une part, les partis politiques structurés, les syndicats et les personnalités de la société civile tolérés par l'État et, d'autre part, les mouvements spontanés d'en bas ?

Le texte, proposé par A. Allal et V. Geisser, "La Tunisie de l'après-Ben Ali, Les partis politiques à la recherche du "peuple introuvable" ", s'intéresse à cette problématique et avance une analyse fine de la construction du champ politique tunisien, lequel avait été verrouillé par cinquante années de pouvoir dictatorial. Selon les auteurs, la problématique s'articule autour de trois dimensions essentielles, imbriquées les unes dans les autres : « les changements à l'œuvre au sein du syndicalisme tunisien, la permanence du dispositif sécuri- 
taire d'ancien régime et les difficultés économiques que connaît le pays ». Les auteurs estiment qu'il est top tôt pour dresser un bilan objectif de ces trois aspects de la vie tunisienne et qu'il n'y a aucune garantie pour une véritable politisation des partis et l'élimination de tous les réseaux et enclaves sécuritaires d'ancien régime.

\section{L'armée et l'État}

Comment peut-on expliquer la coexistence entre un acteur institutionnel de la coercition, l'armée, et les mouvements contestataires ? L'armée, symbole de la violence physique et soutien fondamental des gouvernants des deux régimes prend ses distances vis-à-vis $\mathrm{du}$ « Prince » tout en se rangeant derrière le peuple pour faire figure de libératrice. Les jeunes l'ont saisi très vite et ont souvent envoyé cette parole à l'armée : «Vous êtes de notre côté ». Cette attitude contraste à la fois avec son rôle révolutionnaire paramilitaire des années cinquante - illustré par Nasser en 1952 et Abdol Karim Ghasem en 1958 aussi bien qu'avec son rôle répressif traditionnel ${ }^{7}$.

Cette dualité et la neutralité de l'armée ne nous conduisent-elles pas vers une analyse de la théorie de l'État visant à cerner les particularités de ces gouvernements qui possèdent un système plébiscitaire fortement clientéliste ? Peut-on faire appel au concept de «cartel de pouvoir " ${ }^{8}$ défini par Michel Camau ? Quant à ce phénomène de « cartélisation » du pouvoir disposant à sa tête d'un raïs, comment fonctionne-t-il en période normale et en temps de crise ? Quel scenario est envisageable pour le devenir d'un «État-Cartel »? Comment peut-on passer de l'incertitude révolutionnaire actuelle à une transition démocratique qui exige la mise en place de règles du jeu démocratique?

\section{Le rôle des mouvements islamistes}

L'opinion dominante en Occident a fortement soutenu les deux régimes dictatoriaux contre la menace islamiste et cette tendance n'a cessé d'augmenter après le 11 septembre 2001. L'évolution des mouvances islamistes depuis cette date montre qu'elles font référence à une umma (communauté islamique imaginaire), malmenée par l'Occident et ses alliés du monde arabo-musulman (les régimes arabes corrompus). La défense de l'islam, chez certains musulmans, exige de ses adeptes d'entrer dans un djihad mondialisé contre cette hégémonie occidentale. Or, durant les événements de Tunisie et d'Égypte, les mouvements se sont revendiqués d'une vision sécularisée de l'islam, se focali-

7. Bozarslan H., Réflexions sur les configurations révolutionnaires égyptienne et tunisienne, http://www.mouvements.info/Reflexions-sur-les-configurations.html, consulté, le 12.06.2011.

8. Camau M., «L'exception autoritaire ou l'improbable point d'Archimède de la politique dans le monde arabe » in Picard, E. (ed.) La politique dans le monde arabe, Armand Colin, Paris, 2006, pp. 29-53. 
sant sur la dignité personnelle du citoyen et sur les droits de l'homme. Vontils vers une rupture avec la vision djihadiste de l'islam ? Un modèle d'islam à la turque, à l'opposé d'un islam radical, serait-il envisageable dans ces pays ? L'islam est-il conciliable avec une tradition révolutionnaire ? D’après certains historiens « il y a une longue histoire de soulèvements populaires qui ont conduit à des changements de sultan, voire de dynastie. La pensée politique musulmane reconnaît le droit à la révolte contre un pouvoir injuste » ${ }^{9}$ car le prophète Mahomet a été le seul, dans les religions monothéistes, qui ait pu concrétiser sa pensée politique, à Médine essentiellement, et fonder un véritable modèle social. Ce qui est remarquable dans cette politisation de l'islam dans les mouvements tunisien et égyptien, c'est que, pour la première fois, les idées libérales de l'Europe n'ont pas été imposées d'en haut, comme l'avaient fait le shâh d'Iran ou Atatürk en Turquie, mais qu'elles sont plutôt venues d'en bas tout en s'adaptant à l'islam. Et tout cela surgit paradoxalement dans un contexte particulier où, depuis une dizaine d'années, l'Occident connaît une vague d'islamophobie qui met le vieux continent en décalage avec le monde musulman, lequel se montre explicitement plus ouvert, plus enclin aux idées et aux valeurs des sociétés occidentales.

\section{Les acteurs, les jeunes apolitiques}

On dit souvent que les jeunes sont apolitiques et qu'ils sont plutôt passionnés par les jeux et l'internet. Les deux révolutions ont une fois de plus démontré le manque de fondement de cette idée reçue. Comment s'effectue pour un individu le passage de la frustration apathique à l'intervention collective dans l'espace public ? Comment peut-on analyser le geste du marchand ambulant Sidi Bouzid qui s'est immolé pour devenir le symbole d'un réveil foudroyant ? Le concept du « charisme occasionnel » chez certains humains placés dans une situation extrême peut-il expliquer le geste spontané du jeune Tunisien ? Comment peut-on saisir la continuité d'une identité entre la « soumission » et la « rébellion » 10 ?

\section{La diaspora et le flux migratoire}

Dans certaines expériences révolutionnaires du passé, notamment la révolution russe d'octobre 191711 ou la révolution iranienne de 1979 12, les transformations politiques ont provoqué des vagues de migrations vers les pays démocratiques. Quelles seront les conséquences des flux migratoires tunisien et égyptien dans l'espace européen ? Le concept de l'envahissement de l'Europe par ces diasporas, comme le disent certains médias occidentaux, est-

9. Laurens H., entretien avec le journal Libération, «Le printemps des rêves arabes », Horssérie, pp. 42-45.

10. Bennani-Chraibi M., Soumis et rebelles, les jeunes au Maroc, Paris, Éditions du CNRS, 1994.

11. Goussef C., L'exil russe, la fabrique du réfugié apatride, Paris, CNRS Éditions, 2008, pp. 1045. 
il réellement fondé ? Quel a pu être le rôle de la diaspora déjà installée en Europe dans les développements des mouvements protestataires?

Voilà les problématiques transversales de ce forum. Nous espérons y revenir plus en détail pour un numéro spécial ultérieur.

En guise de mini conclusion de ce forum, je termine cette introduction au Printemps arabe en insistant sur l'effondrement de trois idées-reçues, qui sont des stéréotypes dominants en Occident. Se nourrissant d'une analyse substantialiste, toutes trois stigmatisaient les peuples arabes et la formule du philosophe Pierre Hassner les a résumées en trois principes : « celui du choc des civilisations, celui du monde arabe privé de politique et celui de la stabilité supérieure des régimes autoritaires $» 13$.

12. Vahabi N., La migration iranienne en Belgique, une diaspora par défaut, Paris, L'Harmattan, 2011, pp. 65-70.

13. Hassner P., Libération, «Le printemps des rêves arabes », Hors-série, p. 2. 